\title{
Impact of serum omentin-1 levels on cardiac prognosis in patients with heart failure
}

Taro Narumi, Tetsu Watanabe*, Shinpei Kadowaki, Daisuke Kinoshita, Miyuki Yokoyama, Yuki Honda, Yoichiro Otaki, Satoshi Nishiyama, Hiroki Takahashi, Takanori Arimoto, Tetsuro Shishido, Takuya Miyamoto and Isao Kubota

\begin{abstract}
Background: Various adipokines are reported to be associated with the development of heart failure (HF) through insulin resistance and chronic inflammation. Omentin-1 is a novel adipokine and is associated with incident coronary artery disease. However, it remains unclear whether serum omentin-1 levels are associated with cardiac prognosis in patients with HF.

Methods: We measured serum omentin-1 levels at admission in 136 consecutive patients with HF, and 20 control subjects without signs of significant heart disease. We prospectively followed patients with HF to endpoints of cardiac death or re-hospitalization for worsening HF.

Results: Serum omentin-1 levels were markedly lower in HF patients with cardiac events compared with to without. The patients who were in New York Heart Association (NYHA) functional class IV showed significantly lower serum omentin-1 levels compared to those in class II and III, whereas serum omentin-1 levels did not correlate with serum brain natriuretic peptide levels $(r=0.217, P=0.011)$. We divided the HF patients into three groups based on the tertiles of serum omentin-1 level (low T1, middle T2, and high T3). Multivariate Cox hazard analysis showed that the lowest serum omentin-1 level (T1) was independently associated with cardiac events after adjustment for confounding factors (hazard ratio 5.78, 95\% confidence interval 1.20-12.79). We divided the HF patients into two groups according to the median serum omentin-1 levels. Kaplan-Meier analysis revealed that the patients with low serum omentin-1 levels had a higher risk of cardiac events compared with those with high serum omentin-1 levels (log-rank test $p<0.001$ ).
\end{abstract}

Conclusion: Decreased serum omentin-1 levels were associated with a poor cardiac outcome in patients with HF.

Keywords: Omentin-1, Heart failure, Prognosis

\section{Background}

Heart failure (HF) remain a major cause of death worldwide and has a poor prognosis despite advances in treatment [1]. Adipocytokines, such as tumor necrosis factor-alpha, interleukin-6, and plasminogen activator inhibitor-1, play a crucial role in the development of cardiovascular diseases through insulin resistance and chronic inflammation [2-5]. Adipokines, such as adiponectin, are also reported to have anti-inflammatory, anti-oxidant, and anti-apoptotic properties, and are decreased in patients with cardiovascular disease [6-9]. There has been a move to clarify the causal relationship between various adipokines and cardiovascular disease $[10,11]$.

\footnotetext{
* Correspondence: tewatana@med.id.yamagata-u.ac.jp

Department of Cardiology, Pulmonology, and Nephrology, Yamagata University School of Medicine, 2-2-2 lida-nishi, Yamagata 990-9585, Japan
}

Omentin-1 is a novel adipokine whose serum levels are decreased in obese individuals, and is associated with insulin resistance [12-16]. Omentin-1 has been suggested to play a beneficial role in preventing atherosclerosis $[17,18]$, however, it remains unclear whether serum omentin-1 levels are associated with clinical outcome in patients with HF.

The purpose of this study was to clarify the impact of serum omentin-1 levels on cardiac prognosis in patients with HF.

\section{Methods}

Study population

We enrolled 136 consecutive patients who were admitted to the Yamagata University Hospital for treatment of worsening HF, diagnosis and pathophysiological investigations, 
or for therapeutic evaluation of HF. We also enrolled 20 control subjects without signs of significant heart disease.

A diagnosis of HF was based on a history of dyspnea and symptoms of exercise intolerance followed by pulmonary congestion, pleural effusion, or left ventricular enlargement by chest X-ray or echocardiography $[19,20]$. Control subjects were excluded if they had significant coronary artery disease, systolic and diastolic dysfunction, valvular heart disease, or myocardial hypertrophy on echocardiography [21]. All patients gave written informed consent prior to their participation, and the protocol was approved by the institution's Human Investigation Committee. The procedures were performed in accordance with the Helsinki Declaration.

\section{Measurement of serum omentin-1 and brain natriuretic peptide levels}

Blood samples were drawn at admission and centrifuged at $2,500 \mathrm{~g}$ for 15 minutes at $4^{\circ} \mathrm{C}$ within 30 minutes of collection. The serum was stored at $-80^{\circ} \mathrm{C}$ until analysis. Serum omentin-1 concentrations were measured with a sandwich enzyme-linked immunosorbent assay (ELISA, Immuno-Biological Laboratories CO., Ltd., Gunma, Japan), according to the manufacturer's instructions [22,23]. The serum omentin-1 levels were measured in duplicate by an investigator unaware of the associated patients' characteristics. Serum brain natriuretic peptide (BNP) concentrations were measured using a commercially available specific radio-immuno assay for human BNP (Shiono RIA BNP assay kit, Shionogi \& Co., Ltd., Tokyo, Japan) [24].

\section{Endpoints and follow-up}

The patients were prospectively followed for a median duration of $399 \pm 378$ days. The end points were cardiac death, including death due to progressive HF, myocardial infarction, stroke and sudden cardiac death, and re-hospitalization for worsening HF. Sudden cardiac death was defined as death without definite premonitory symptoms or signs, and was confirmed by the attending physician. Two cardiologists who were blinded to the blood biomarker data reviewed the medical records and conducted telephone interviews to survey the incidence of cardiovascular events.

\section{Statistical analysis}

Data are presented as the mean \pm standard deviation (SD). The Mann-Whitney U-test was used when the data were not distributed normally. If the data were not distributed normally, they were presented as medians with an interquartile range. The unpaired Student's t-test and the chi-square test were used for comparisons of continuous and categorical variables, respectively. Comparison of data among three groups was performed by the Kruskal-Wallis test. Uni- and multivariate analyses with Cox proportional hazard regression were used to determine significant predictors of cardiovascular events. Cumulative overall and event-free survival rates were computed using the Kaplan-Meier method and were compared using the logrank test. We calculated the net reclassification improvement (NRI) and the integrated discrimination improvement (IDI) to measure the quantity of improvement for the correct reclassification and sensitivity according to the addition of serum omentin-1 levels to the prediction model [25]. NRI and IDI are new statistical measures to assess and quantify the improvement in risk prediction offered by a new marker. A $P$ value $<0.05$ was considered statistically significant. All statistical analyses were performed with a standard statistical program package (JMP version 10; SAS Institute, Cary, North Carolina, USA), and the R-3.0.2 with additional packages (Rcmdr, Epi, pROC, and PredictABEL).

\section{Results}

\section{Comparison between patients with and without heart failure}

The patients with HF had a lower BMI and left ventricular ejection fraction, and lower serum total cholesterol, triglyceride levels, and higher serum BNP levels compared with control subjects (Table 1).

\section{Comparison between HF patients with and without cardiac events}

There were 59 cardiac events including 17 deaths and 32 re-hospitalizations in patients with HF during the follow-up period (Table 2). The patients who experienced cardiac events were in a more severe New York Heart Association (NYHA) functional class, and had a lower estimated glomerular filtration rate, lower left ventricular ejection fraction, higher left ventricular end-diastolic diameter, and higher serum BNP levels compared with those who did not. Moreover, patients with cardiac events showed markedly lower serum omentin-1 levels compared with those without (Figure 1). There were no significant differences in etiologies of HF between patients with and without cardiac events (Table 2).

\section{Serum omentin- 1 levels and HF severity}

The patients who were in NYHA functional class IV showed significantly lower serum omentin-1 levels compared to those in class II and III $(\mathrm{P}=0.029$ vs. class II and $\mathrm{P}=0.041$ vs. class III, Figure 2A). On the other hand, serum omentin-1 levels were not significantly different between the patients who were in NYHA functional class II and III ( $\mathrm{P}=0.582)$. Furthermore, there was no relationship between the serum omentin-1 levels and the serum BNP levels $(r=0.217$, Figure 2B). 
Table 1 Baseline clinical characteristics

\begin{tabular}{lccc}
\hline & $\begin{array}{c}\text { Control } \\
(\mathbf{n = 2 0})\end{array}$ & $\begin{array}{c}\text { Heart failure } \\
(\mathbf{n = 1 3 6 )}\end{array}$ & $\begin{array}{c}\boldsymbol{P} \\
\text { value }\end{array}$ \\
\hline Age, years & $65 \pm 16$ & $72 \pm 12$ & 0.034 \\
Male, n (\%) & $11(55)$ & $76(56)$ & 0.941 \\
$\begin{array}{l}\text { NYHA functional class, } \\
\text { II/IIIIV }\end{array}$ & - & $71 / 46 / 19$ & - \\
\end{tabular}

Etiology, n (\%)

Dilated cardiomyopathy

$29(21)$

Valvular heart disease

38 (28)

Ischemic heart disease

Hypertensive heart disease

Hypertrophic cardiomyopathy

Others

$30(22)$

$14(10)$

$9(7)$

$16(12)$

Presentation profile

Systolic pressure, $\mathrm{mmHg}$

Diastolic pressure, $\mathrm{mmHg}$

Body mass index, $\mathrm{kg} / \mathrm{m}^{2}$

eGFR, $\mathrm{ml} / \mathrm{min} / 1.73 \mathrm{~m}^{2}$

Blood biomarkers

Albumin, $\mathrm{g} / \mathrm{dl}$

Total cholesterol, mg/dl

Triglyceride, $\mathrm{mg} / \mathrm{dl}$

LDLc, mg/dl

$\mathrm{HDLC}, \mathrm{mg} / \mathrm{dl}$

hsCRP, mg/dl (IQR)

BNP, pg/ml (IQR)

Omentin-1, ng/ml (IQR)

Echocardiographic data

LV end-diastolic diameter, $\mathrm{mm}$

LV ejection fraction, \%

Medications, $\mathrm{n}(\%)$

ACE inhibitors and/or ARBs

$\beta$ blockers

$\begin{array}{ccc}119 \pm 22 & 117 \pm 18 & 0.772 \\ 77 \pm 10 & 74 \pm 10 & 0.209 \\ 23.3 \pm 3.4 & 21.7 \pm 3.9 & 0.049 \\ 70 \pm 24 & 62 \pm 26 & 0.197\end{array}$

$\begin{array}{ccc}3.8 \pm 0.5 & 3.5 \pm 0.6 & 0.091 \\ 185 \pm 34 & 166 \pm 39 & 0.042 \\ 143 \pm 96 & 91 \pm 47 & <0.001 \\ 111 \pm 28 & 100 \pm 37 & 0.213 \\ 52 \pm 15 & 53 \pm 23 & 0.831 \\ 0.121 & 0.198 & 0.279 \\ (0.040-0.551) & (0.064-0.606) & \end{array}$

$82(50-152) \quad 484(215-1251)<0.001$ $494(351-630) \quad 305(35-473) \quad 0.035$

Statins

$53 \pm 8 \quad 55 \pm 11 \quad 0.438$

$65 \pm 9 \quad 50 \pm 18 \quad<0.001$

$15(75) \quad 102(75) \quad 0.999$

$15(75) \quad 103(76) \quad 0.943$

$10(50) \quad 51(38) \quad 0.321$
Ca channel blockers

$37(27) \quad 0.778$

Data are presented as mean $\pm S D$ or $\%$ unless otherwise indicated; $A C E$, angiotensin-converting enzyme; $\mathrm{ARB}$, angiotensin receptor blocker; $\mathrm{BNP}$, brain natriuretic peptide; BUN, Blood urea nitrogen; eGFR, estimated glomerular filtration rate; $\mathrm{HDLc}$, high density lipoprotein cholesterol; hsCRP, high-sensitivity C-reactive protein; IQR, interquartile range; LDLc, low density lipoprotein cholesterol; LV, left ventricular; NYHA, New York Heart Association.

\section{Association between serum omentin-1 levels and cardiac events}

We divided patients with HF into three groups according to the tertiles of serum omentin-1 levels. Multivariate Cox hazard analysis showed that the lowest serum omentin-1 levels (T1) were independently associated with cardiac events after adjustment for age, gender, NYHA functional
Table 2 Comparison of patients with or without cardiac event

\begin{tabular}{lccc}
\hline & $\begin{array}{c}\text { Event (-) } \\
(\mathbf{n = 7 7 )}\end{array}$ & $\begin{array}{c}\text { Event }(+) \\
(\mathbf{n}=59)\end{array}$ & $\begin{array}{c}\boldsymbol{P} \\
\text { value }\end{array}$ \\
\hline Age, years & $71 \pm 10$ & $72 \pm 14$ & 0.687 \\
Male, n (\%) & $40(52)$ & $36(61)$ & 0.480 \\
NYHA functional class, II/II/IV & $49 / 21 / 6$ & $22 / 25 / 13$ & 0.005 \\
Etiology, n (\%) & & & 0.348 \\
Dilated cardiomyopathy & $12(16)$ & $17(29)$ & - \\
Valvular heart disease & $24(31)$ & $14(24)$ & - \\
Ischemic heart disease & $17(22)$ & $13(22)$ & - \\
Hypertensive heart disease & $8(10)$ & $6(10)$ & - \\
Hypertrophic cardiomyopathy & $6(8)$ & $3(5)$ & - \\
Others & $10(13)$ & $6(10)$ & -
\end{tabular}

Presentation profile

Systolic pressure, $\mathrm{mmHg}$

Diastolic pressure, $\mathrm{mmHg}$

Body mass index, $\mathrm{kg} / \mathrm{m}^{2}$

eGFR, $\mathrm{ml} / \mathrm{min} / 1.73 \mathrm{~m}^{2}$

Blood biomarkers

Albumin, $\mathrm{g} / \mathrm{dl}$

Total cholesterol, mg/dl

Triglyceride, $\mathrm{mg} / \mathrm{dl}$

LDLc, mg/dl

$\mathrm{HDLC}, \mathrm{mg} / \mathrm{dl}$

hsCRP, mg/dl (IQR)

\begin{tabular}{|c|c|c|}
\hline $116 \pm 18$ & $118 \pm 19$ & 0.598 \\
\hline $73 \pm 9$ & $74 \pm 11$ & 0.780 \\
\hline $22.0 \pm 4.4$ & $21.4 \pm 3.3$ & 0.413 \\
\hline $66 \pm 27$ & $57 \pm 24$ & 0.046 \\
\hline $3.5 \pm 0.6$ & $3.5 \pm 0.6$ & 0.539 \\
\hline $169 \pm 37$ & $163 \pm 42$ & 0.398 \\
\hline $97 \pm 48$ & $84 \pm 45$ & 0.132 \\
\hline $101 \pm 37$ & $99 \pm 37$ & 0.731 \\
\hline $55 \pm 29$ & $52 \pm 12$ & 0.437 \\
\hline $\begin{array}{c}0.174 \\
(0.058-0.330)\end{array}$ & $\begin{array}{c}0.267 \\
(0.073-0.722)\end{array}$ & 0.308 \\
\hline $453(248-1249)$ & $512(169-1255)$ & 0.049 \\
\hline $479(323-661)$ & $139(57-402)$ & $<0.00$ \\
\hline $53 \pm 10$ & $57 \pm 10$ & 0.011 \\
\hline $56 \pm 17$ & $45 \pm 16$ & $<0.0$ \\
\hline $54(70)$ & $48(81)$ & 0.134 \\
\hline $52(68)$ & $51(86)$ & 0.029 \\
\hline $23(30)$ & $28(47)$ & 0.460 \\
\hline $29(3)$ & $8(14)$ & 0.964 \\
\hline
\end{tabular}

BNP, pg/ml (IQR)

Omentin-1, ng/ml (IQR)

Echocardiographic data

LV end-diastolic diameter, $\mathrm{mm}$

LV ejection fraction, \%

Medications, n (\%)

ACE inhibitors and/or ARBs

$\beta$ blockers

Statins

Ca channel blockers

8 (14)

Data are presented as mean $\pm S D$ or $\%$ unless otherwise indicated; $A C E$,
angiotensin-converting enzyme; $A R B$, angiotensin receptor blocker; BNP, brain natriuretic peptide; BUN, Blood urea nitrogen; eGFR, estimated glomerular filtration rate; $\mathrm{HDLc}$, high density lipoprotein cholesterol; hsCRP, high-sensitivity C-reactive protein; IQR, interquartile range; LDLc, low density lipoprotein cholesterol; LV, left ventricular; NYHA, New York Heart Association.

class, left ventricular ejection fraction, and serum brain natriuretic peptide levels (hazard ratio 5.65, 95\% confidence interval 2.61-12.20; Figure 3, Table 3). We divided the patients into two groups according to the median serum omentin-1 levels. Kaplan-Meier analysis revealed that the patients with low serum omentin-1 levels had a 


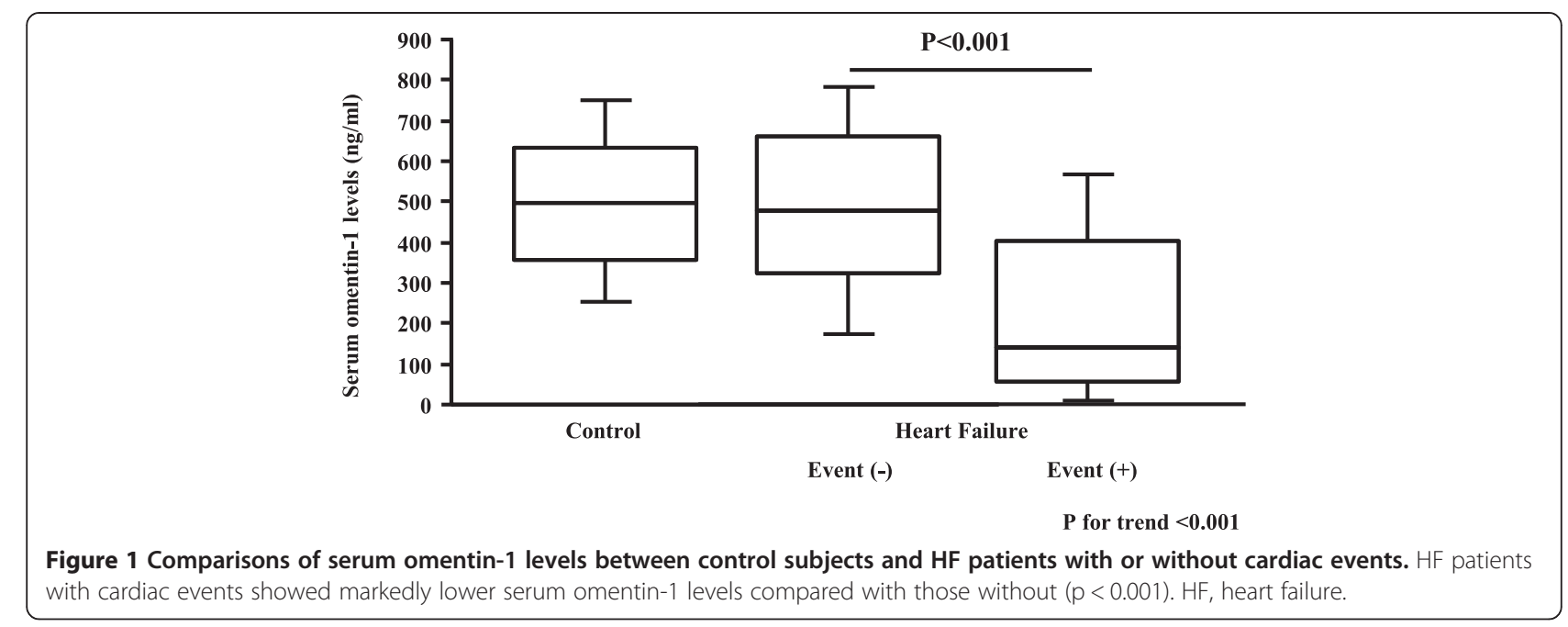

higher risk of cardiac events compared to those with high serum omentin-1 levels (log-rank test $\mathrm{p}<0.001$, Figure 4).

\section{Net reclassification improvement and integrated discrimination improvement}

To measure the quantity of improvement for the correct reclassification and sensitivity according to the addition of serum omentin-1 levels to the prediction model, we calculated the NRI and the IDI. The inclusion of serum omentin-1 levels in the prediction model (includes age, gender, NYHA functional class, left ventricular ejection fraction, and serum BNP levels) for the prediction of cardiac events, improved the NRI and IDI values, suggesting effective reclassification and discrimination (Table 4).

\section{Discussion}

The present study demonstrated that decreased serum omentin-1 levels predicted cardiac events in patients with
HF. Serum omentin-1 level appears to be a novel prognostic marker for the risk stratification of patients with HF.

Various types of adipocytokines are reported to be a predictor of unfavorable cardiac outcomes in patients with HF [26]. In addition to their roles as predictors of cardiac outcome, a variety of adipocytokines have been associated with the development of HF through insulin resistance and chronic inflammation [14,27-29]. Serum adiponectin levels are reported to be correlated with BNP levels, and are associated with HF severity and unfavorable outcomes in patients with HF [30,31]. Adiponectin has been suggested to play a role in the prevention of cardiovascular diseases via its anti-inflammatory, anti-oxidant, and antiapoptotic properties [6-9]. Recently, reports have shown several adipokines to have beneficial effects on cardiovascular diseases [32-34]. However, the precise role of these adipokines remains unclear.

Omentin-1 is a $38 \mathrm{kDa}$ novel adipokine identified in 2004 from visceral adipose tissue $[12,13]$. Shibata et al. reported

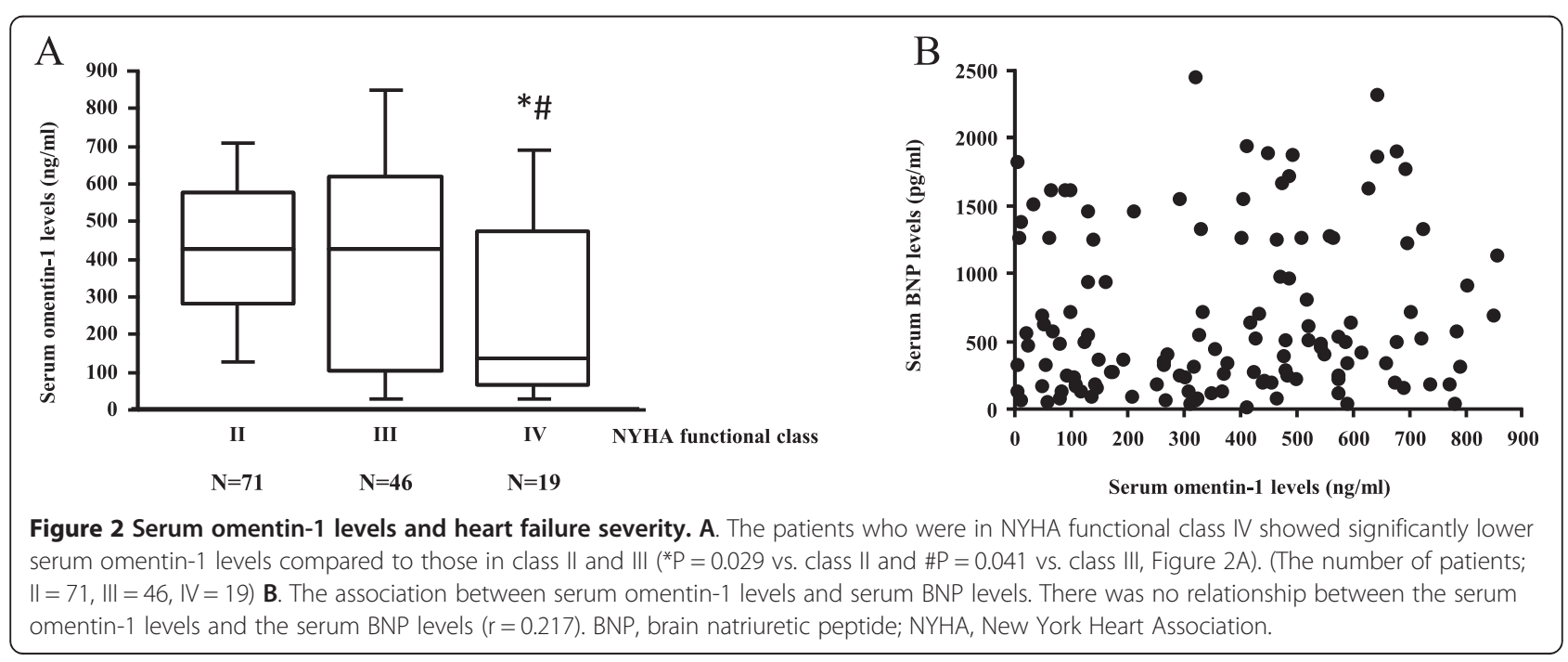




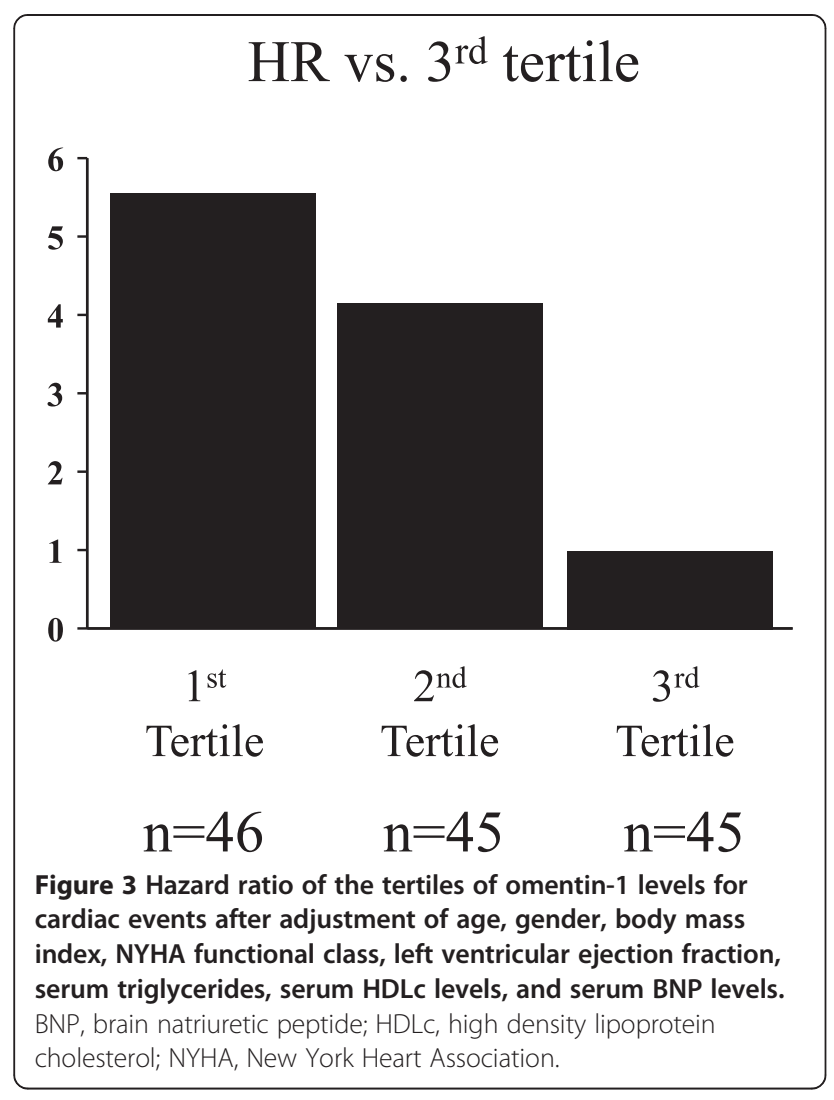

that decreased plasma omentin-1 levels predict the prevalence of coronary artery disease [18]. Yang et al. reported that omentin-1 enhances insulin-stimulated glucose uptake in human adipocytes and may regulate insulin sensitivity [13]. Yamawaki et al. reported that omentin-1 modulates vascular function and attenuates cyclooxygenase-2 expression and c-jun N-terminal kinase (JNK) activation in cytokine-stimulated endothelial cells [35,36]. These studies all suggest that omentin-1 may improve insulin resistance and suppress vascular inflammation. Interestingly, Pan et al. suggested that omentin-1 expression and production are

Table 3 Univariate and multivariate analyses for cardiac events

\begin{tabular}{lcccccccc}
\hline & \multicolumn{3}{c}{ Univariate Analysis } & & \multicolumn{3}{c}{ Multivariate Analysis } \\
\cline { 2 - 3 } & HR & $\mathbf{9 5 \%} \mathbf{C l}$ & $\begin{array}{c}\boldsymbol{P} \\
\text { value }\end{array}$ & & $\begin{array}{c}\text { Adjusted } \\
\text { HR }^{*}\end{array}$ & $\mathbf{9 5 \%} \mathbf{C l}$ & $\begin{array}{c}\boldsymbol{P} \\
\text { value }\end{array}$ \\
\hline Omentin-1 & & & & & & & & \\
T3 & 1 & Reference & Reference & 1 & Reference & Reference \\
T2 & 5.56 & $2.85-10.87$ & $<0.001$ & & 4.15 & $2.03-8.47$ & $<0.001$ \\
T1 & 6.29 & $1.30-$ & $<0.001$ & & 5.65 & $2.61-12.20$ & $<0.001$
\end{tabular}

*Adjusted HR after adjustment for age, gender, body mass index, NYHA functional class, left ventricular ejection fraction, serum triglycerides, serum HDLc levels, and serum BNP levels.

$\mathrm{BNP}$, brain natriuretic peptide; $\mathrm{Cl}$, confidence interval; $\mathrm{HDLC}$, high density lipoprotein cholesterol; HR, hazard ratio; NYHA, New York Heart Association $\mathrm{SD}$, standard deviation.

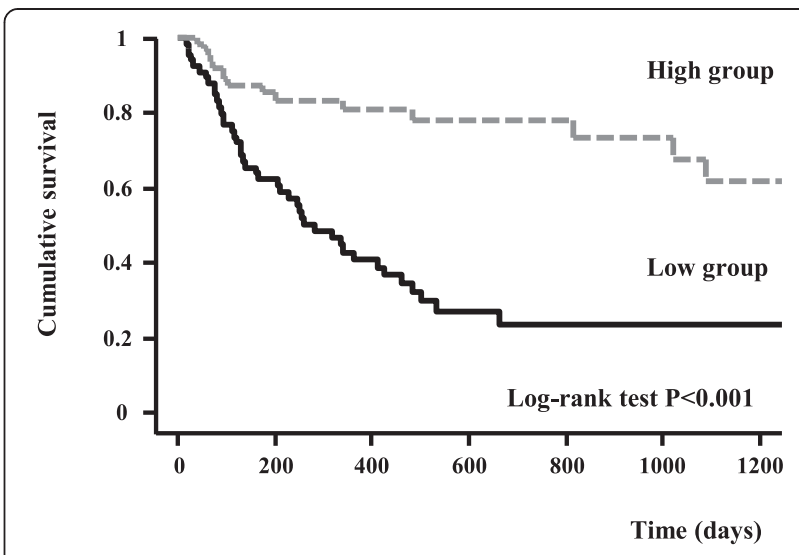

Figure 4 Kaplan-Meier analysis. The patients with low serum omentin-1 levels had a higher risk of cardiac events compared to those with high serum omentin-1 levels (log-rank test $p<0.001)$.

decreased with elevated inflammatory adipokines, such as tumor necrosis factor-alpha and interleukin-6, in patients with impaired glucose intolerance and newly diagnosed type 2 diabetes mellitus [37].

Unlike to adiponectin, serum omentin-1 was reported to decrease with chronic inflammation and oxidative stress in patients with HF. The bioactivity of omentin-1 appears multifaceted and remains to be fully defined. The present study showed no correlation between serum omentin-1 and BNP levels unlike adiponectin [30], suggesting that these markers indicate different features of the pathophysiological process of HF. Serum omentin-1 levels may represent a promising biomarker for cardiac prognosis, irrespective of serum BNP levels. The inclusion of serum omentin-1 levels in the prediction model (includes age, gender, NYHA functional class, left ventricular ejection fraction, and serum BNP levels) for the prediction of cardiac events, improved the NRI and IDI values, suggesting effective reclassification and discrimination.

The present study has certain limitations. Firstly, the sample size was relatively small and it was a single center study. Nonetheless, there was a significant relationship between serum omentin-1 levels and cardiac events. In addition, the inclusion of serum omentin-1 levels in the

Table 4 Statistics for model fit and improvement with addition of serum omentin-1 level predicted on the prediction of cardiac events

\begin{tabular}{llcc}
\hline & $\begin{array}{c}\text { Prediction } \\
\text { model }\end{array}$ & $\begin{array}{c}\text { Prediction model + } \\
\text { omentin-1 }\end{array}$ & P value \\
\hline $\mathrm{NRI}(95 \% \mathrm{Cl})$ & Reference & $0.375(0.129-0.620)$ & 0.002 \\
$\mathrm{IDI}(95 \% \mathrm{Cl})$ & Reference & $0.149(0.087-0.211)$ & $<0.001$
\end{tabular}

Prediction model includes age, gender, NYHA functional class, left ventricular ejection fraction, and serum BNP levels.

$\mathrm{BNP}$, brain natriuretic peptide; $\mathrm{Cl}$, confidence interval; IDI, integrated discrimination improvement; NRI, net reclassification improvement; NYHA, New York Heart Association. 
prediction model with conventional risk factors, including serum BNP levels, for the prediction of cardiac events, improved the NRI and IDI values. Secondly, there were no data for other adipocytokines. Further study is needed to clarify the association between serum omentin-1 and other adipocytokines in a large HF population.

In conclusion, decreased serum omentin-1 levels were associated with cardiac events in patients with HF, irrespective of serum BNP levels. Serum omentin-1 level appears to represent a novel prognostic marker for the risk stratification of patients with HF.

\section{Abbreviations}

BMI: Body mass index; BNP: Brain natriuretic peptide; eGFR: Estimated glomerular filtration rate; ELISA: Sandwich enzyme-linked immunosorbent assay; HF: Heart failure; IDI: Integrated discrimination improvement; NRI: Net reclassification improvement; NYHA: New York heart association;

SD: Standard deviation.

\section{Competing interests}

The authors report that there is no duality of interest associated with this manuscript.

\section{Authors' contributions}

TN, TW and IK contributed to discussions about study design and data analyses. SK, DK, MY, YO, and YH conceived and carried out experiments. TN and TW participated in the interpretation of the results and the writing of the manuscript. SN, HT, TA, TS, and TM helped with data collection. All authors have read and approved the final manuscript.

\section{Acknowledgements}

The authors would like to express their gratitude to the staff at the Department of Cardiology, Pulmonology, and Nephrology, Yamagata University School of Medicine, Yamagata, Japan for their kind cooperation while conducting this study. Research described in this manuscript was supported in part by a grant-in-aid for Scientific Research (No. 24591033) from the Ministry of Education Culture, Sport, Science, and Technology.

Received: 4 March 2014 Accepted: 15 April 2014

Published: 23 April 2014

\section{Reference}

1. Jessup M, Brozena S: Heart failure. New Engl J Med 2003, 348(20):2007-2018.

2. Bradham WS, Ormseth MJ, Oeser A, Solus JF, Gebretsadik T, Shintani A, Stein CM: Insulin Resistance is Associated with Increased Concentrations of NT-proBNP in Rheumatoid Arthritis: IL-6 as a Potential Mediator. Inflammation 2014, DOI:10.1007/s10753-013-9799-4.

3. Mohler ER 3rd, Sorensen LC, Ghali JK, Schocken DD, Willis PW, Bowers JA, Cropp AB, Pressler ML: Role of cytokines in the mechanism of action of amlodipine: the PRAISE Heart Failure Trial. Prospective Randomized Amlodipine Survival Evaluation. J Am Coll Cardiol 1997, 30(1):35-41.

4. Aispuru GR, Clavier MM, Cardone AJ, Gilberto DO, Barousse AP: Thrombotic biomarkers and left ventricle characteristics as short-term predictors of thrombotic events in patients hospitalized for acute decompensated heart failure. Eur J Intern Med 2012, 23(6):545-551.

5. Kim HM, Lee BW, Song YM, Kim WJ, Chang HJ, Choi DH, Yu HT, Kang E, Cha BS, Lee HC: Potential association between coronary artery disease and the inflammatory biomarker YKL-40 in asymptomatic patients with type 2 diabetes mellitus. Cardiovasc Diabetol 2012, 11(1):84

6. Ouchi N, Kihara S, Arita Y, Maeda K, Kuriyama H, Okamoto Y, Hotta K Nishida M, Takahashi M, Nakamura T, Yamashita S, Funahashi T, Matsuzawa Y: Novel modulator for endothelial adhesion molecules: adipocyte-derived plasma protein adiponectin. Circulation 1999, 100(25):2473-2476.

7. von Eynatten M, Schneider JG, Humpert PM, Kreuzer J, Kuecherer H, Katus HA, Nawroth PP, Dugi KA: Serum adiponectin levels are an independent predictor of the extent of coronary artery disease in men. J Am Coll Cardiol 2006, 47(10):2124-2126
8. Sun $Y$, Chen $X$ : Effect of adiponectin on apoptosis: proapoptosis or antiapoptosis? BioFactors (Oxford, England) 2010, 36(3):179-186.

9. Zhang Y, Wang XL, Zhao J, Wang YJ, Lau WB, Yuan YX, Gao EH, Koch WJ, Ma XL: Adiponectin inhibits oxidative/nitrative stress during myocardial ischemia and reperfusion via PKA signaling. Am J Physiol Endocrinol Metab 2013, 305(12):E1436-1443.

10. Agra RM, Teijeira-Fernandez E, Pascual-Figal D, Sanchez-Mas J, FernandezTrasancos A, Gonzalez-Juanatey JR, Eiras S: Adiponectin and p53 mRNA in epicardial and subcutaneous fat from heart failure patients. Eur J Clin Invest 2014, 44(1):29-37.

11. Karas MG, Benkeser D, Arnold AM, Bartz TM, Djousse L, Mukamal KJ, IX JH, Zieman SJ, Siscovick DS, Tracy RP, Mantzoros CS, Gottdiener JS, deFilippi CR, Kizer JR: Relations of Plasma Total and High-Molecular-Weight Adiponectin to New-Onset Heart Failure in Adults $>/=65$ Years of Age (from the Cardiovascular Health Study). Am J Cardiol 2013, 113(2):328-334.

12. Schaffler A, Neumeier M, Herfarth H, Furst A, Scholmerich J, Buchler C: Genomic structure of human omentin, a new adipocytokine expressed in omental adipose tissue. Biochim Biophys Acta 2005, 1732(1-3):96-102.

13. Yang RZ, Lee MJ, Hu H, Pray J, Wu HB, Hansen BC, Shuldiner AR, Fried SK, MCLenithan JC, Gong DW: Identification of omentin as a novel depotspecific adipokine in human adipose tissue: possible role in modulating insulin action. Am J Physiol Endocrinol Metab 2006, 290(6):E1253-1261.

14. de Souza Batista CM, Yang RZ, Lee MJ, Glynn NM, Yu DZ, Pray J, Ndubuizu K, Patil S, Schwartz A, Kligman M, Fried SK, Gong DW, Shuldiner AR, Pollin TI, McLenithan JC: Omentin plasma levels and gene expression are decreased in obesity. Diabetes 2007, 56(6):1655-1661.

15. Tan BK, Adya R, Farhatullah S, Lewandowski KC, O'Hare P, Lehnert H, Randeva HS: Omentin-1, a novel adipokine, is decreased in overweight insulin-resistant women with polycystic ovary syndrome: ex vivo and in vivo regulation of omentin-1 by insulin and glucose. Diabetes 2008, 57(4):801-808

16. Liu R, Wang X, Bu P: Omentin-1 is associated with carotid atherosclerosis in patients with metabolic syndrome. Diabetes Res Clin Pract 2011, 93(1):21-25.

17. Yoo HJ, Hwang SY, Hong HC, Choi HY, Yang SJ, Seo JA, Kim SG, Kim NH, Choi KM, Choi DS, Baik SH: Association of circulating omentin-1 level with arterial stiffness and carotid plaque in type 2 diabetes. Cardiovasc Diabetol 2011, 10:103.

18. Shibata R, Ouchi N, Kikuchi R, Takahashi R, Takeshita K, Kataoka Y, Ohashi K, Ikeda N, Kihara S, Murohara T: Circulating omentin is associated with coronary artery disease in men. Atherosclerosis 2011, 219(2):811-814.

19. McKee PA, Castelli WP, McNamara PM, Kannel WB: The natural history of congestive heart failure: the Framingham study. N Engl J Med 1971, 285(26):1441-1446.

20. Jessup M, Abraham WT, Casey DE, Feldman AM, Francis GS, Ganiats TG, Konstam MA, Mancini DM, Rahko PS, Silver MA, Stevenson LW, Yancy CW: 2009 focused update: ACCF/AHA Guidelines for the Diagnosis and Management of Heart Failure in Adults: a report of the American College of Cardiology Foundation/American Heart Association Task Force on Practice Guidelines: developed in collaboration with the International Society for Heart and Lung Transplantation. Circulation 2009, 119(14):1977-2016.

21. Funayama A, Shishido T, Netsu S, Ishino M, Sasaki T, Katoh S, Takahashi H, Arimoto T, Miyamoto T, Nitobe J, Watanabe T, Kubota I: Serum pregnancyassociated plasma protein $a$ in patients with heart failure. $J$ Card Fail 2011, 17(10):819-826.

22. Tsuji S, Tsuura Y, Morohoshi T, Shinohara T, Oshita F, Yamada K, Kameda Y, Ohtsu T, Nakamura Y, Miyagi Y: Secretion of intelectin-1 from malignant pleural mesothelioma into pleural effusion. Br J Canc 2010, 103(4):517-523.

23. Tsuji S, Uehori J, Matsumoto M, Suzuki Y, Matsuhisa A, Toyoshima K, Seya T: Human intelectin is a novel soluble lectin that recognizes galactofuranose in carbohydrate chains of bacterial cell wall. J Biol Chem 2001, 276(26):23456-23463.

24. Narumi T, Arimoto T, Funayama A, Kadowaki S, Otaki Y, Nishiyama S, Takahashi H, Shishido T, Miyashita T, Miyamoto T, Watanabe T, Kubota I: The prognostic importance of objective nutritional indexes in patients with chronic heart failure. J Cardiol 2013, 62(5):307-313.

25. Pencina MJ, D'Agostino RB Sr, D'Agostino RB Jr, Vasan RS: Evaluating the added predictive ability of a new marker: from area under the ROC curve to reclassification and beyond. Stat Med 2008, 27(2):157-172. discussion 207-112 
26. Frankel DS, Vasan RS, D'Agostino RB Sr, Benjamin EJ, Levy D, Wang TJ, Meigs JB: Resistin, adiponectin, and risk of heart failure the Framingham offspring study. J Am Coll Cardiol 2009, 53(9):754-762.

27. Shimizu I, Yoshida Y, Katsuno T, Tateno K, Okada S, Moriya J, Yokoyama M, Nojima A, Ito T, Zechner R, Komuro I, Kobayashi Y, Minamino T: p53induced adipose tissue inflammation is critically involved in the development of insulin resistance in heart failure. Cell metabolism 2012, 15(1):51-64.

28. Lago F, Dieguez C, Gomez-Reino J, Gualillo O: Adipokines as emerging mediators of immune response and inflammation. Nat Clin Pract Rheumatol 2007, 3(12):716-724.

29. Aroor $\mathrm{AR}$, Mandavia $\mathrm{CH}$, Sowers JR: Insulin resistance and heart failure: molecular mechanisms. Heart Fail Clin 2012, 8(4):609-617.

30. Tsutamoto T, Tanaka T, Sakai H, Ishikawa C, Fujii M, Yamamoto T, Horie M: Total and high molecular weight adiponectin, haemodynamics, and mortality in patients with chronic heart failure. Eur Heart J 2007, 28(14):1723-1730.

31. Baldasseroni S, Antenore A, Di Serio C, Orso F, Lonetto G, Bartoli N, Foschini A Marella A, Pratesi A, Scarantino S: Adiponectin, diabetes and ischemic heart failure: a challenging relationship. Cardiovasc Diabetol 2012, 11:151.

32. Adeghate E: Visfatin: structure, function and relation to diabetes mellitus and other dysfunctions. Curr Med Chem 2008, 15(18):1851-1862.

33. Hida K, Wada J, Eguchi J, Zhang H, Baba M, Seida A, Hashimoto I, Okada T, Yasuhara A, Nakatsuka A, Shikata K, Hourai S, Futami J, Watanabe E, Matsuki Y, Hiramatsu R, Akagi S, Makino H, Kanwar YS: Visceral adipose tissue-derived serine protease inhibitor: a unique insulin-sensitizing adipocytokine in obesity. Proc Natl Acad Sci U S A 2005, 102(30):10610-10615.

34. Christou GA, Tselepis AD, Kiortsis DN: The metabolic role of retinol binding protein 4: an update. Horm Metab Res 2012, 44(1):6-14.

35. Yamawaki $H$, Tsubaki N, Mukohda M, Okada M, Hara Y: Omentin, a novel adipokine, induces vasodilation in rat isolated blood vessels. Biochem Biophys Res Commun 2010, 393(4):668-672.

36. Yamawaki H, Kuramoto J, Kameshima S, Usui T, Okada M, Hara Y: Omentin, a novel adipocytokine inhibits TNF-induced vascular inflammation in human endothelial cells. Biochem Biophys Res Commun 2011, 408(2):339-343.

37. Pan HY, Guo L, Li Q: Changes of serum omentin-1 levels in normal subjects and in patients with impaired glucose regulation and with newly diagnosed and untreated type 2 diabetes. Diabetes Res Clin Pract 2010, 88(1):29-33.

doi:10.1186/1475-2840-13-84

Cite this article as: Narumi et al:: Impact of serum omentin-1 levels on cardiac prognosis in patients with heart failure. Cardiovascular Diabetology 2014 13:84.

\section{Submit your next manuscript to BioMed Central and take full advantage of:}

- Convenient online submission

- Thorough peer review

- No space constraints or color figure charges

- Immediate publication on acceptance

- Inclusion in PubMed, CAS, Scopus and Google Scholar

- Research which is freely available for redistribution 